\title{
Effective and precise adenine base editing in mouse zygotes
}

\section{Dear Editor,}

Many human genetic diseases are caused by pathogenic single nucleotide mutations. Animal models are often used to study these diseases where the pathogenic point mutations are created and/or corrected through gene editing (e.g., the CRISPR/Cas9 system) (Komor et al., 2017; Liang et al., 2017). CRISPR/Cas9-mediated gene editing depends on DNA double-strand breaks (DSBs), which can be of low efficiency and lead to indels and off-target cleavage (Kim et al., 2016). We and others have shown that base editors (BEs) may represent an attractive alternative for disease mouse model generation (Liang et al., 2017; Kim et al., 2017). Compared to CRISPR/ Cas9, cytidine base editors (CBEs) can generate $C \cdot G$ to $T \cdot A$ mutations in mouse zygotes without activating DSB repair pathways (Liang et al., 2017; Kim et al., 2017; Komor et al., 2016). In addition, CBEs showed much lower off-targets than CRISPR/Cas9 (Kim et al., 2017), making the editing process potentially safer and more controllable. Recently, adenine base editors (ABEs) that were developed from the tRNAspecific adenosine deaminase (TADA) of Escherichia coli were also reported (Gaudelli et al., 2017). As a RNA-guided programmable adenine deaminase, $A B E$ can catalyze the conversion of $A$ to $I$. Following DNA replication, base $I$ is replaced by $G$, resulting in $A \cdot T$ to $G \cdot C$ conversion (Gaudelli et al., 2017; Hu et al., 2018). The development of ABEs has clearly expanded the editing capacity and application of BEs. Here, we tested whether ABEs could effectively generate disease mouse models, and found high efficiency by ABEs in producing edited mouse zygotes and mice with single-nucleotide substitutions.

Unlike CBEs that can generate premature stop codons with C-T conversion (TAG, TAA or TGA), ABEs cannot produce a new stop codon to disrupt gene function via A-G conversion. We therefore targeted mRNA splice sites in order to induce gene dysfunction. Since mammalian mRNA splicing requires a $5^{\prime} \mathrm{GU}$ donor and a $3^{\prime} \mathrm{AG}$ acceptor at intron-exon junctions, ABEs can block mRNA splicing and hence inactivate gene function by converting splice donors and acceptors to GC and GG. We named this strategy ABEinduced mRNA splicing defect (Al-MAST).

We first used ABE7.10 to target the mouse Tyr gene, whose dysfunction results in albinism in mice (Zhang et al.,
2016). A gRNA was designed to target the splice donor at exon 3 of the Tyr gene, which is also predicted to be an ideal site for $A B E$. We then injected both ABE7.10 mRNA and the gRNA into mouse zygotes (Fig. S1A). Of the 20 embryos harvested $48 \mathrm{~h}$ later, 9 were edited (45.0\%) with efficiencies ranging from $11.2 \%$ to $24.6 \%$ (Fig. S1B-D). In addition, 106 injected zygotes were transplanted into pseudopregnant mothers. Among the 23 pups obtained, 13 (56.5\%) showed A-to-G editing with conversion frequencies of $14.6 \%-48.1 \%$ (Figs. S1B and S2), attesting to the feasibility of Al-MAST in generating point mutations in mice.

It should be noted that we did not obtain any white-coated F0 mice, likely due to insufficient A-to-G conversion rate at the splice donor site. However, when the T1-12 F0 mouse was mated with homozygous $T y r$ mutant (c.655G>T, $p$. E219X) C57BL/6J mice (Liang et al., 2017), 2/5 (40.0\%) pups were albino (Fig. S3A). Sanger sequencing results indicated that the 2 albino pups were compound heterozygous for both the ABE target site and Tyr site (c.655G>T, $p$. E219X) (Fig. S3B), lending support to Tyr gene dysfunction as a result of $A \cdot T$ to $G \cdot C$ conversion at the splice donor of exon 3. Furthermore, analysis of RNAs extracted from the skin of these compound heterozygous mice found significant reduction of correctly spliced Tyr mRNAs compared with $\mathrm{Ty}^{\mathrm{F} 219 \mathrm{X} /+}$ mice (Fig. S3C and S3D). Both Tyr $\mathrm{F}^{\mathrm{E} 19 \mathrm{X} /+}$ and TyF ${ }^{\text {E219X/E219X }}$ mice showed obvious reduction of Tyr mRNA, indicating that $T y r^{\mathrm{E} 219 \mathrm{X}}$ mutant RNA is subjected to degradation by nonsense-mediated mRNA decay (NMD). These data demonstrate that AI-MAST is capable of inducing mRNA splicing defects. However, whether phenotypes associated with mRNA splicing defects can be observed in F0 mice remains unknown.

To further explore one-step generation of disease mouse models using ABEs, we designed two gRNAs that targeted the splice sites at exons 61 and 66 of Dmd (Fig. 1A). These two sites were chosen because Dunchenne muscular dystrophy (DMD) remains a progressive neuromuscular degenerative disorder with no effective treatment. The largest in the human genome with 79 exons and $2.4 \mathrm{Mb}$ long, the human $D M D$ gene has recorded thousands of mutations (2,898 in the UMD-DMD database for DMD patients), including insertions, deletions, duplications and point mutations. At least 158 splice site mutations have been identified 


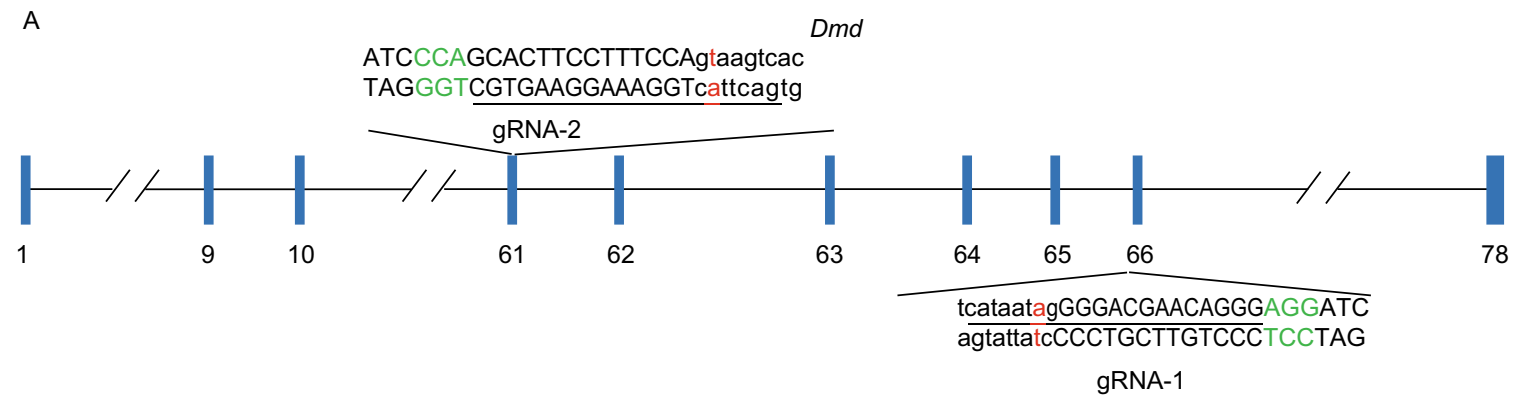

B

\begin{tabular}{|c|c|c|c|c|c|c|}
\hline $\begin{array}{l}\text { gRNA } \\
\text { group }\end{array}$ & $\begin{array}{l}\text { Genotyped } \\
\text { embryos (\#) }\end{array}$ & $\begin{array}{c}\text { Mutant } \\
\text { embryos (\#) }\end{array}$ & $\begin{array}{l}\text { Transferred } \\
\text { embryos (\#) }\end{array}$ & $\begin{array}{c}\text { Total } \\
\text { pups } \\
(\#)\end{array}$ & $\begin{array}{c}\text { Mutant } \\
\text { pups } \\
(\#)\end{array}$ & $\begin{array}{c}\text { Mutant } \\
\text { pups with } \\
>95.0 \% \\
\text { efficiency } \\
\text { (\#) }\end{array}$ \\
\hline 1 & 18 & $\begin{array}{c}15 \\
(83.3 \%)\end{array}$ & 270 & $\begin{array}{c}67 \\
(24.8 \%)\end{array}$ & $\begin{array}{c}47 \\
(70.1 \%)\end{array}$ & $\begin{array}{c}14 \\
(20.9 \%)\end{array}$ \\
\hline 2 & 17 & $\begin{array}{c}9 \\
(52.9 \%)\end{array}$ & 122 & $\begin{array}{c}26 \\
(21.3 \%)\end{array}$ & $\begin{array}{c}11 \\
(42.3 \%)\end{array}$ & $\begin{array}{c}1 \\
(3.8 \%)\end{array}$ \\
\hline
\end{tabular}

gRNA-1

gRNA-2

C

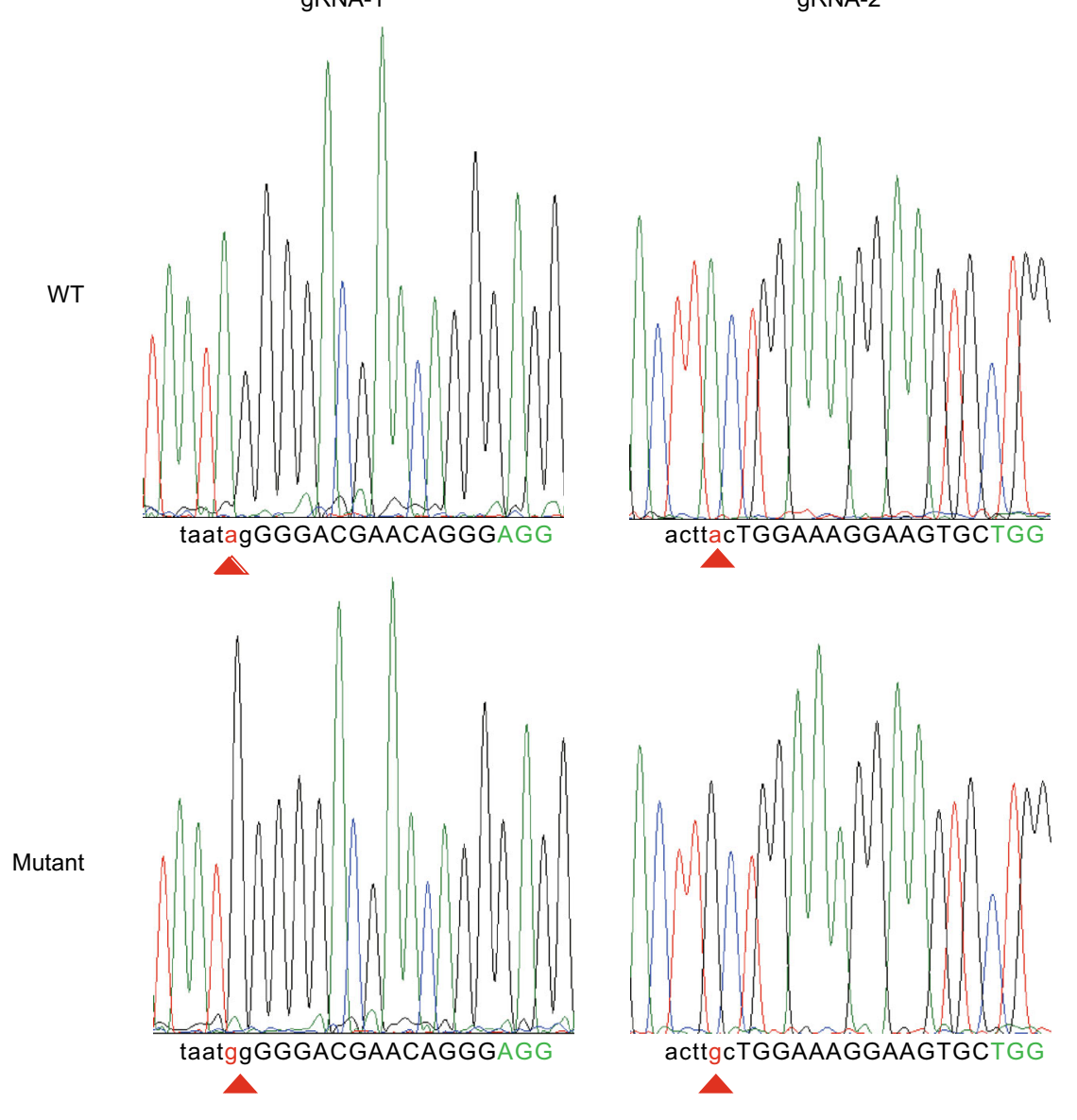


Figure 1. Efficient targeting of the mouse Dmd gene by adenine base editors (ABEs). (A) Schematic representation of the two gRNA target sites in the Dmd gene locus. Exon-intron boundary sequences (both strands) are shown with exon sequences capitalized and intron sequences in lower case. The gRNA target sequence is underlined, with PAM in green and the adenine being mutated in red. (B) The number of injected and transplanted embryos and subsequent pup information for each gRNA group are listed in the table. (C) Representative Sanger sequencing chromatograms of PCR amplicons spanning each gRNA target site from wild-type (WT) vs. mutant mice (D1-12 and D2-22 for group gRNA-1 and gRNA-2 respectively). Red triangle marks the targeted/mutated adenine. (D) PCR amplicons spanning the gRNA-1 target site from the F0 newborns were analyzed by deep sequencing. Exons and introns are in capital and lower case letters respectively. Base substitutions, red. PAM, green. The frequency of each mutant allele within individual pups is listed on the right. (E) PCR amplicons spanning the gRNA-2 target site from the F0 newborns were analyzed by deep sequencing.

thus far, including the splice donor of exon 61 (c.9163 $+1 \mathrm{G}>\mathrm{A}$, GU-to-AU; c. $9163+2 \mathrm{~T}>\mathrm{G}$, GU-to-GG) and splice acceptor of exon 66 (c.9564-2A>T, AG-to-TG) (http://www. umd.be/DMD/4DACTION/W_DMDT1/9). Exons 61 and 66 are highly conserved between human and mouse, both at the genomic DNA (>91\% similarity) and protein $(100 \%)$ sequence level. With gRNA-1 and gRNA-2, $15 / 18$ (83.3\%)

D

$$
\begin{array}{lll}
\text { WT } & \text { tcataatagGGGACGAACAGGGAGGATC } & \text { Ratio }(\%) \\
\text { D1-1 } & \text { tcatatggGGGACGAACAGGGAGGATC } & 95.9 \\
& \text { tcatagtggGGACGAACAGGGAGGATC } & 2.7 \\
\text { D1-2 } & \text { tcataatggGGGACGAACAGGGAGGATC } & 99.2 \\
\text { D1-7 } & \text { tcataatggGGGACGAACAGGGAGGATC } & 96.5 \\
\text { D1-12 } & \text { tcataatggGGGACGAACAGGGAGGATC } & 94.6 \\
& \text { tcatagtggGGACGAACAGGGAGGATC } & 2.3 \\
\text { D1-16 } & \text { tcataatggGGACGAACAGGGAGGATC } & 98.2 \\
\text { D1-18 } & \text { tcataatggGGACGAACAGGGAGGATC } & 99.4 \\
\text { D1-19 } & \text { tcataatggGGGACGAACAGGGAGGATC } & 76.8 \\
& \text { tcatagtggGGGACGAACAGGGAGGATC } & 23.0 \\
\text { D1-20 } & \text { tcataatggGGGACGAACAGGGAGGATC } & 99.4 \\
\text { D1-24 } & \text { tcataatggGGGACGAACAGGGAGGATC } & 98.6 \\
\text { D1-25 } & \text { tcataatggGGACGAACAGGGAGGATC } & 99.2 \\
\text { D1-26 } & \text { tcataatggGGACGAACAGGGAGGATC } & 97.0 \\
& \text { tcatagtggGGACGAACAGGGAGGATC } & 2.2 \\
\text { D1-27 } & \text { tcataatggGGGACGAACAGGGAGGATC } & 99.0 \\
\text { D1-28 } & \text { tcataatggGGGACGAACAGGGAGGATC } & 98.8 \\
\text { D1-38 } & \text { tcataatggGGGACGAACAGGGAGGATC } & 97.2
\end{array}
$$

Figure 1. continued. and 9/17 (52.9\%) embryos were respectively edited (Fig. 1B). Allelic mutation frequencies in these embryos ranged from $10.1 \%$ to $88.7 \%$ (Fig. S4). Following transplantation of the gRNA-1 (270) and gRNA-2 (122) injected zygotes, $70.1 \%$ (47/67) pups were edited with varying allelic mutation frequency for gRNA-1, and $42.3 \%$ (11/26) pups were edited for gRNA-2 with a slightly lower allelic mutation frequency compared to gRNA-1 (Fig. 1B-E). While allelic mutation frequency differs among F0 mice, a total of 15 F0 mice were obtained that exhibited $>95.0 \%$ frequency. Of the 67 F0 mice from group gRNA-1, 14 were edited with $>95.0 \%$ efficiency (20.9\%) (Fig. 1B). Remarkably, 5 of these 14 mice showed a rate of $>99.0 \%$ (Fig. 1D). These 5 mice may in fact be pure mutant mice without any wild-type alleles, considering that error rates of deep sequencing may run up to $1 \%$ (Liang et al., 2017). In addition, one F0 mouse was edited with $98.0 \%$ efficiency from group gRNA-2 (Fig. 1E). These results combinedly suggest high editing efficiency of $A B E s$ and the Al-MAST strategy in mouse zygotes.

We next sought to determine whether the ABE-induced Dmd splice site mutant mice would display DMD phenotypes. First, we quantified correctly spliced Dmd mRNAs by qPCR analysis of RNAs isolated from the quadriceps and hearts of mutant mice and their wild-type (WT) littermates (Fig. S5A). Correctly spliced Dmd mRNAs decreased significantly $(>90 \%)$ in edited mice, indicating highly efficient base editing by AI-MAST (Figs. 2A and S5B). In addition, we were also able to detect incorrectly spliced transcripts that

E WT gtgacttacTGGAAAGGAAGTGCTGGGAT Ratio (\%)

D2-2 gtgacttgcTGGAAAGGAGTGCTGGGAT 84.4

D2-3 gtgacttgcTGGAAAGGAAGTGCTGGGAT 33.3

D2-4 gtgacttgcTGGAAAGGAAGTGCTGGGAT 13.4

D2-17 gtgacttgcTGGAAAGGAAGTGCTGGGAT 58.5

D2-18 gtgacttgcTGGAAAGGAAGTGCTGGGAT 84.3

D2-19 gtgacttgc TGGAAAGGAAGTGCTGGGAT 52.7

D2-22 gtgacttgcTGGAAAGGAAGTGCTGGGAT 98.0

D2-23 gtgacttgc TGGAAAGGAGTGCTGGGAT 28.2

D2-24 gtgacttgcTGGAAAGGAAGTGCTGGGAT 77.5 
A

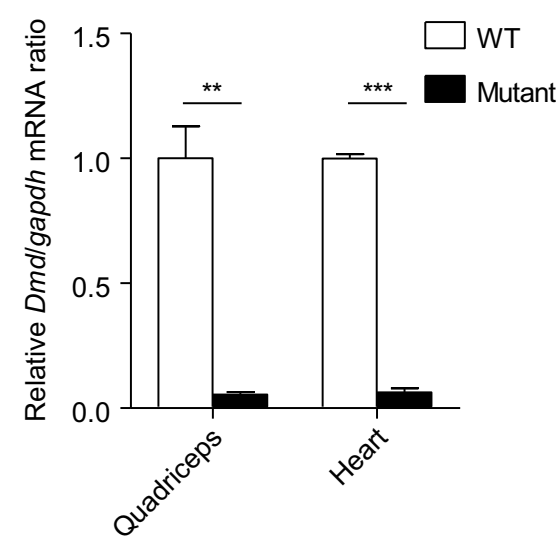

B

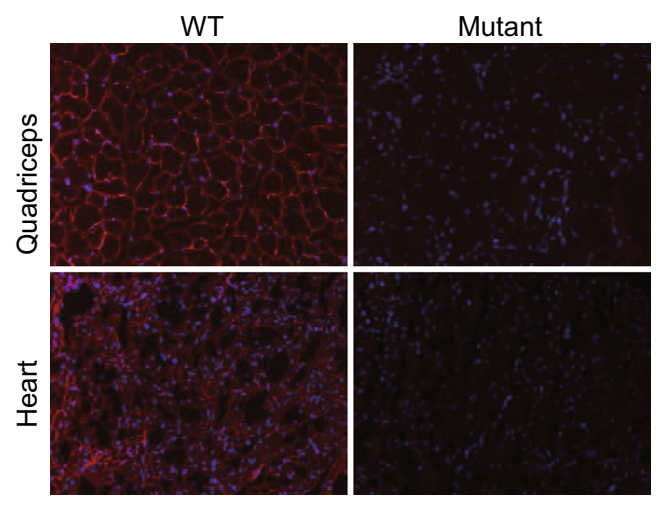

C

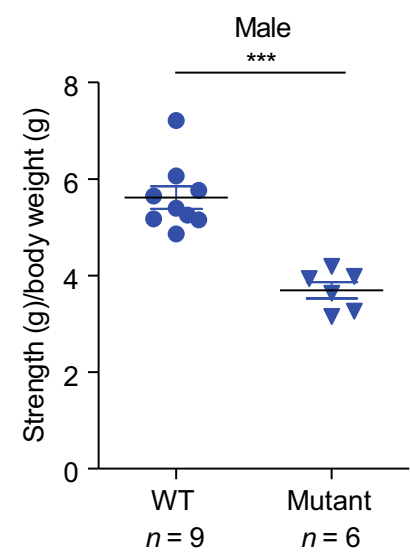

D

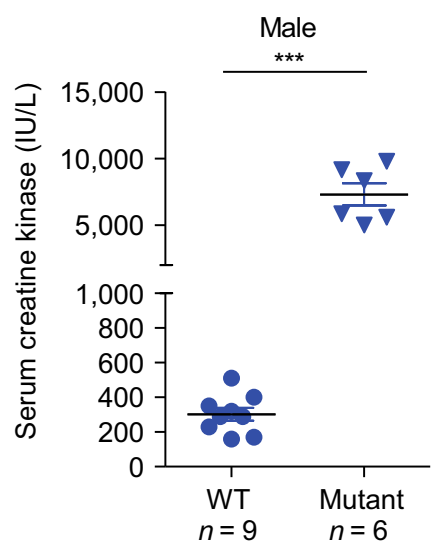

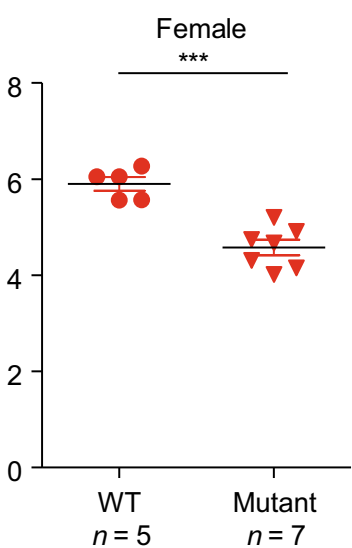

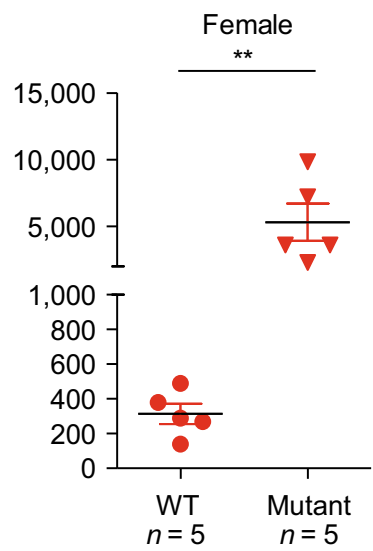

Figure 2. One-step generation of DMD mice by ABE-induced mRNA splicing defect strategy (AI-MAST). (A) qPCR was carried out using RNAs extracted from the quadriceps and hearts of WT (D1-4, D1-14, D1-31) and mutant mice (D1-18, D1-25, D1-27) to quantify correctly spliced $D m d$ mRNAs. Data are presented as mean $\pm \operatorname{SEM}(n=3) .{ }^{\star \star} P<0.01 .{ }^{* \star} P<0.001$. Statistical significance was determined using the two-tailed Student's $t$-test. (B) Immunofluorescence staining of Dmd in WT and mutant mice from (A). Representative images from pups D1-4 (WT) and D1-18 (mutant) were shown $(n=3)$. (C) Forelimb grip strength of 4-5 week old male and female mice from the Dmd gRNA-1 group was assessed using a force transducer. ${ }^{* * *} P<0.001$. Statistical significance was determined using the two-tailed $t$-test. (D) Serum creatine kinase levels in 4-5 week old male and female mice from the Dmd gRNA-1 group were determined. ${ }^{* *} P<0.01$, and ${ }^{* * *} P<0.001$. Statistical significance was determined using the two-tailed $t$-test. (E and $\mathrm{F}$ ) Analysis of human disease splice site mutations that may be modeled by ABE7.10 (E) and xCas9(3.7)-ABE7.10 (F). 


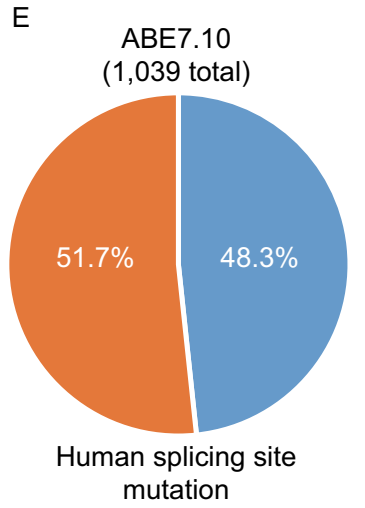

$\mathrm{F}$

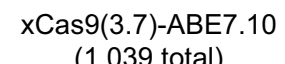
( 1,039 total)

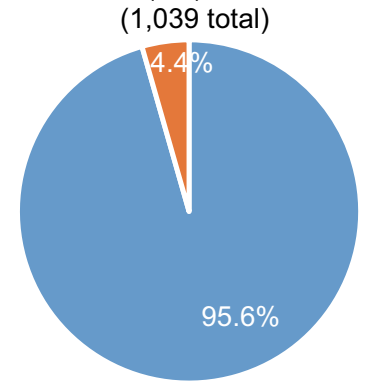

Human splicing site mutation

AI-MAST untargetable

Al-MAST targetable

Figure 2. continued.

appeared to have used cryptic splice sites (Fig. S5C and S5D). Consistent with the drastic reduction in properly spliced mRNAs, the Dmd gene product (Dystrophin protein) was also nearly depleted in edited mice as assayed by immunostaining (Fig. 2B).

Dystrophin is necessary for muscle fiber strength, the absence of which results in muscle weakness. In humans, splicing defects in the DMD gene can cause Duchenne muscular dystrophy with severe symptoms including muscle fatigability and myocardial fibrosis (Birnkrant et al., 2018). Similarly, we also observed significant decreases in the forelimb grip strength of both male and female mutant mice (Figs. 2C, S6 and Table S1). Creatine kinase (CK) activity is a widely used marker in the investigation of skeletal muscle diseases (Birnkrant et al., 2018). In line with their muscle weakness, serum creatine kinase (CK) levels in these mutant mice were substantially elevated as well compared with WT controls, similar to muscular dystrophy phenotypes observed in Dmd mouse models (Fig. 2D and Table S2). When mated with WT mice, the A-to-G Dmd mutations in the mutant mice could be stably passed down to their progenies (Fig. S7). These data indicate that AI-MAST is suitable to establishing mouse models for human diseases in one step.

Off-target effects are a well-known problem of canonical CRISPR-Cas9 editing tools. To examine the rate of off-target deamination in $\mathrm{F} 0$ mice, we selected 10 mutant mice from each gRNA group for deep sequencing. For the top 5 predicted off-target sites of each gRNA (based on sequence similarity), no off-target deamination was found (Tables S35). Roughly $2 / 22$ (9.1\%) CBE-edited embryos and $3 / 57$ (5.3\%) CBE-edited F0 mice were found to contain alleles with indels (Liang et al., 2017; Kim et al., 2017). In comparison, we did not find any indels in ABE-edited embryos (33) or F0 mice (71). In addition, we found only A-to-G conversions, but no A-to-C/T conversions, which is in agreement with ABEs' observed improved product purity in

human cells (Gaudelli et al., 2017). Taken together, our data demonstrate that $A B E s$ can efficiently and precisely convert base $A$ to $G$ in mouse embryos and represent a high-fidelity tool in generating point mutation mouse models.

We estimate that $-79.4 \%$ and $87.3 \%$ respectively of mouse and human protein-coding genes may be targeted by the AlMAST strategy (Fig. S8A, S8B and Tables S6-7), suggesting broad applicability of Al-MAST in making gene deficiency mouse models and human cell lines. The recently developed xCas9 (3.7)-ABE7.10, an ABE variant with a broader PAM preference $\left(5^{\prime}-\mathrm{NGN}-3^{\prime}, 5^{\prime}-\mathrm{GAA}-3^{\prime}, 5^{\prime}-\mathrm{GAT}-3^{\prime}\right.$ and $5^{\prime}$-CAA-3') (Hu et al., 2018) should further expand the target scope of our AI-MAST strategy (Fig. S8C, S8D and Tables S8-9). In humans, $\sim 10 \%$ of pathogenic mutations in all Mendelian diseases comprise of splice site mutations (Faustino and Cooper, 2003), Our AI-MAST strategy therefore should prove particularly attractive in the generation of relevant animal models and the investigation of human diseases caused by splice-site defects. In-depth analysis revealed that $48.3 \%(517 / 1,039)$ of the human pathogenic mutations at splice sites can be generated by ABE7.10 (Fig. 2E and Table S10), and $95.6 \%$ can be generated by $x$ Cas 9(3.7)-ABE7.10 (Fig. 2F and Table S11). In addition, we also found some conserved splice site mutations in human and mouse that can be generated by either ABE7.10 (64) or xCas9 (3.7)-ABE7.10 (163) (Tables S12 and S13). Working with human cell and mouse models of these mutation sites has the best chance of probing disease biology and developing possible new therapeutics.

While our manuscript was under review, two independent groups reported using ABEs to generate mouse models and repair disease mutations in adult mouse (Ryu et al., 2018; Liu et al., 2018). Our study together with the others not only highlight the fidelity and efficiency of $A B E s$ in inducing $A \cdot T$ to $G \cdot C$ conversion, but also demonstrate their potential ease and versatility in generating disease models as well as correcting disease mutations in animal and human embryos (Liang et al., 2017).

\section{FOOTNOTES}

This work was supported by the National Key R\&D Program of China (2017YFC1001901 and 2017YFA0102801), the Science and Technology Planning Project of Guangdong Province (2015B020228002), the National Natural Science Foundation of China (Grant Nos. 91640119, 31671540, 81330055 and 31601196), the Natural Science Foundation of Guangdong Province (2016A030310206 and 2014A030312011), the Guangzhou Science and Technology Project (201605030012 and 201707010085) and China Postdoctoral Science Foundation (2017M622862).

Puping Liang, Hongwei Sun, Xiya Zhang, Xiaowei Xie, Jinran Zhang, Yaofu Bai, Xueling Ouyang, Shengyao Zhi, Yuanyan Xiong, Wenbin Ma, Dan Liu, Junjiu Huang and Zhou Songyang declare that they have no conflict of interest. All institutional and national guidelines for the care and use of laboratory animals were followed.

Z. Songyang, J. Huang, and P. Liang designed the research. P. Liang, H. Sun, X. Zhang, X. Xie, J. Zhang, Y. Bai, X. Ouyang, S. Zhi, Y. Xiong, W. Ma performed the experiments. D. Liu revised 
manuscript. J. Huang and Z. Songyang supervised the research. All authors discussed the results and commented on the manuscript.

Puping Liang ${ }^{1,2 \bowtie}$, Hongwei Sun ${ }^{1}$, Xiya Zhang ${ }^{1}$, Xiaowei Xie ${ }^{1}$, Jinran Zhang ${ }^{1}$, Yaofu Bai ${ }^{1}$, Xueling Ouyang ${ }^{1}$, Shengyao Zhi ${ }^{1}$, Yuanyan Xiong ${ }^{1}$, Wenbin $\mathrm{Ma}^{1}$, Dan Liu ${ }^{4}$, Junjiu Huang ${ }^{1,2,3 凶}$, Zhou Songyang ${ }^{1,2,3,4 \bowtie}$

${ }^{1}$ Key Laboratory of Gene Engineering of the Ministry of Education, Guangzhou Key Laboratory of Healthy Aging Research and SYSU-BCM Joint Research Center, School of Life Sciences, Sun Yat-sen University, Guangzhou 510275, China

2 Key Laboratory of Reproductive Medicine of Guangdong Province, School of Life Sciences and the First Affiliated Hospital, Sun Yatsen University, Guangzhou 510275, China

${ }^{3}$ State Key Laboratory of Ophthalmology, Zhongshan Ophthalmic Center, Sun Yat-sen University, Guangzhou 510060, China

${ }^{4}$ Verna and Marrs Mclean Department of Biochemistry and Molecular Biology, Baylor College of Medicine, One Baylor Plaza, Houston, TX 77030, USA

$\bowtie$ Correspondence: liangpp5@mail.sysu.edu.cn (P. Liang), hjunjiu@mail.sysu.edu.cn (J. Huang), songyanz@mail.sysu.edu.cn (Z. Songyang)

\section{OPEN ACCESS}

This article is distributed under the terms of the Creative Commons Attribution 4.0 International License (http://creativecommons.org/ licenses/by/4.0/), which permits unrestricted use, distribution, and reproduction in any medium, provided you give appropriate credit to the original author(s) and the source, provide a link to the Creative Commons license, and indicate if changes were made.

\section{REFERENCES}

Birnkrant DJ, Bushby K, Bann CM, Apkon SD, Blackwell A, Brumbaugh D, Case LE, Clemens PR, Hadjiyannakis S, Pandya $S$ et al (2018) Diagnosis and management of Duchenne muscular dystrophy, part 1: diagnosis, and neuromuscular, rehabilitation, endocrine, and gastrointestinal and nutritional management. Lancet Neurol 17(3):251-267
Faustino NA, Cooper TA (2003) Pre-mRNA splicing and human disease. Genes Dev 17:419-437

Gaudelli NM, Komor AC, Rees HA, Packer MS, Badran AH, Bryson DI, Liu DR (2017) Programmable base editing of $A^{*} T$ to $G^{*} C$ in genomic DNA without DNA cleavage. Nature 551:464-471

$\mathrm{Hu}$ JH, Miller SM, Geurts MH, Tang W, Chen L, Sun N, Zeina CM, Gao X, Rees HA, Lin Z et al (2018) Evolved Cas9 variants with broad PAM compatibility and high DNA specificity. Nature 556:57-63

Kim D, Kim S, Kim S, Park J, Kim JS (2016) Genome-wide target specificities of CRISPR-Cas9 nucleases revealed by multiplex Digenome-seq. Genome Res 26:406-415

Kim K, Ryu SM, Kim ST, Baek G, Kim D, Lim K, Chung E, Kim S, Kim JS (2017a) Highly efficient RNA-guided base editing in mouse embryos. Nat Biotechnol 35:435-437

Kim D, Lim K, Kim ST, Yoon SH, Kim K, Ryu SM, Kim JS (2017b) Genome-wide target specificities of CRISPR RNA-guided programmable deaminases. Nat Biotechnol 35:475-480

Komor AC, Kim YB, Packer MS, Zuris JA, Liu DR (2016) Programmable editing of a target base in genomic DNA without double-stranded DNA cleavage. Nature 533:420-424

Komor AC, Badran AH, Liu DR (2017) CRISPR-Based technologies for the manipulation of eukaryotic genomes. Cell 169:559

Liang P, Zhang X, Chen Y, Huang J (2017a) Developmental history and application of CRISPR in human disease. J Gene Med. https://doi.org/10.1002/jgm.296

Liang P, Sun H, Sun Y, Zhang X, Xie X, Zhang J, Zhang Z, Chen Y, Ding $C$, Xiong $Y$ et al (2017b) Effective gene editing by highfidelity base editor 2 in mouse zygotes. Protein Cell 8:601-611

Liang P, Ding C, Sun H, Xie X, Xu Y, Zhang X, Sun Y, Xiong Y, Ma W, Liu $Y$ et al (2017c) Correction of beta-thalassemia mutant by base editor in human embryos. Protein Cell 8:811-822

Liu Z, Lu Z, Yang G, Huang S, Li G, Feng S, Liu Y, Li J, Yu W, Zhang $Y$ et al (2018) Efficient generation of mouse models of human diseases via ABE- and BE-mediated base editing. Nat Commun 9:2338

Ryu SM, Koo T, Kim K, Lim K, Baek G, Kim ST, Kim HS, Kim D, Lee $\mathrm{H}$, Chung $\mathrm{E}$ et al (2018) Adenine base editing in mouse embryos and an adult mouse model of Duchenne muscular dystrophy. Nat Biotechnol 36:536-539

Zhang X, Liang P, Ding C, Zhang Z, Zhou J, Xie X, Huang R, Sun Y, Sun $\mathrm{H}$, Zhang J et al (2016) Efficient production of gene-modified mice using Staphylococcus aureus Cas9. Sci Rep 6:32565
Puping Liang, Hongwei Sun, Xiya Zhang and Xiaowei Xie contributed equally to this work.

Electronic supplementary material The online version of this article (https://doi.org/10.1007/s13238-018-0566-z) contains supplementary material, which is available to authorized users. 